\title{
Neue Ansätze zur diagnostischen und therapeutischen Arbeit mit der Konfliktachse der OPD-KJ
}

Inge Seiffge-Krenke

Psychologisches Institut der Universität Mainz

International hat sich in der Kinder- und Jugendpsychiatrie und Psychotherapie seit Anfang der 1990er-Jahre und dem Erscheinen von ICD-10 (International Statistical Classification of Diseases and Related Health Problems) und DSM-IV (Diagnostic and Statistical Manual of Mental Disorders) mit ihrer phänomenologisch-deskriptiven Diagnostik und Symptomfokussierung die Erkenntnis durchgesetzt, dass es relevante Kategorien psychischer Gesundheit und Krankheit auch jenseits des Symptoms gibt (Dilling \& Feyberger, 2012). Auch der Krankheitswandel mit seiner Zunahme an strukturellen Störungen machte eine Ergänzung des klassischen Konfliktmodells als Krankheitsmodell psychodynamischer Provenienz durch eine Strukturpathologie bzw. Traumapathologie notwendig (Seiffge-Krenke, 2020). Es erschien sinnvoll, Konzepte zu entwickeln und empirisch zu erproben, die bei der Einschätzung der Indikation zu verschiedenen Formen der Behandlung, zur Einschätzung der Behandlungsmotivation und zum Finden eines Behandlungsfokus hilfreich sind.

In der Kinder- und Jugendpsychiatrie und Psychotherapie findet die Operationalisierte Psychodynamische Diagnostik (Arbeitskreis OPD-KJ-2, 2020) in den letzten Jahren zunehmend Anwendung. Sie ist eine operationalisierte psychodynamische Diagnostik, die in Anlehnung an die OPD für Erwachsene entwickelt wurde und vier psychodynamische und deskriptive Achsen enthält. In der ersten Achse, den Behandlungsvoraussetzungen werden Leidensdruck und Motivation für eine Behandlung erhoben. Die Achse Beziehung erfasst in der Selbst- und Fremdwahrnehmung anhand beobachtungsnaher Kategorien wie Nähe und Distanz bzw. Wärme und Kontrolle die Qualität der Beziehung zwischen Untersucher_in und Kind. Die Achse Konflikt erfasst sieben zeitlich überdauernde und verinnerlichte Konflikte, die das Leben von Kindern und Jugendlichen einschränken und die Interaktionen färben. Die Achse Struktur erfasst Defizite in der psychischen Struktur, schwerpunktmäßig bezogen auf Bindung, Emotionsregulierung und die Selbst- und Objektwahrnehmung.

Das Instrument wurde seit der Gründung 1996 durch eine interdisziplinär aus Kinder- und Jugendpsychiater_innen, Kinder- und Jugendtherapeut_innen und Entwicklungspsycholog_innen zusammengesetzte Arbeitsgruppe zur Operationalisierung entsprechender Konzepte mehrfach, auch auf der Basis der Ergebnisse von OPD-KJ-Trainings, revidiert, in der Operationalisierung der relevanten psychodynamischen Konstrukte geschärft und liegt gegenwärtig in der dritten Fassung (Arbeitskreis OPD-KJ-2, 2020) vor. Die Besonderheiten dieses diagnostischen Systems sind neben der klaren Ressourcenorientierung die Tatsache, dass Beeinträchtigungen und Ressourcen über verschiedene Lebensbereiche wie Familie, Gleichaltrige/ Freunde und Schule, Körper erfasst werden können, sowie seine Altersnormierung: Die Operationalisierung erfolgt altersgruppenspezifisch und orientiert sich an Piaget. Eingeschätzt wird im Manual anhand der Altersstufen 1 (2-5 Jahre, d.h. das Vorschulkind), 2 (6-12 Jahre, d.h. die mittlere Kindheit) und 3 (13 Jahre und älter, d.h. ab der Pubertät). Die Beschäftigung mit operationalisierten Kriterien für diagnostische Einschätzungen führte zu einem merklichen Zugewinn an Genauigkeit und Differenziertheit der Konstrukte. Es wurde aber auch deutlich, dass die „richtigen Fragen“" gestellt werden müssen, um eine exakte und beobachtungsnahe Einschätzung zu ermöglichen. Dies führte folgerichtig zu einem Leitfaden mit Fragen für die diagnostische Interviewführung (Arbeitskreis OPD$\mathrm{KJ}-2,2020$ ).

Eine der Zielsetzungen der OPD-KJ war es, eine einheitliche Sprache zwischen verschiedenen Betreuer_innen von Kindern und Jugendlichen im ambulanten und stationären Bereich zu vermitteln. Kinder- und Jugendpsychiater_innen und Psychotherapeut_innen haben die Erfahrung gemacht, dass es anhand der von OPD-KJ vorgenommenen 
Operationalisierungen leichter gelingt, Übereinstimmung im Austausch mit Fachkolleg_innen zu erzielen und dennoch die Komplexität psychodynamischer Konzepte zu erhalten. Inzwischen ist die Operationalisierte Psychodynamische Diagnostik im Kindes- und Jugendalter an vielen Psychotherapie-Weiterbildungsinstituten fester Bestandteil der Curricula geworden, und zwar nicht nur an psychodynamisch orientierten Instituten, sondern auch an Instituten, die eine Verhaltenstherapie- oder eine systemische Ausbildung anbieten. Durch die Veröffentlichung des englischen Manuals OPD-CA-2 im Jahr 2017 und die geplante Veröffentlichung des spanischen Manuals in 2022 wurde der Interessentenkreis für die Operationalisierte Psychodynamische Diagnostik noch einmal erheblich erweitert. Die OPD-KJ ist Gegenstand der schriftlichen Prüfung zur Approbation, sie wird für Kassenanträge zur Richtlinienpsychotherapie und in der Qualitätssicherung von psychotherapeutischen Behandlungen eingesetzt.

Nachdem empirische Arbeiten zur Achse Struktur der OPD-KJ bereits 2019 Gegenstand eines Themenheftes der Zeitschrift für Kinder- und Jugendpsychiatrie und Psychotherapie (Heft 47) waren, geht es in diesem Themenheft um Befunde und neuere Ansätze zur Konfliktachse der OPDKJ. Die Konfliktachse erhebt die über die Diagnose bzw. Symptomatik hinausgehenden entwicklungsbehindernden Konflikte und erlaubt damit auch das Finden eines Behandlungsfokus.

In dem vorliegenden Themenheft steht die Konfliktachse der OPD-KJ im Vordergrund, die eine Operationalisierung von sieben psychodynamischen Konflikten und den jeweils zugeordneten Verarbeitungsmodi enthält. Orientiert an psychodynamischen Konzepten wird nach OPD-KJ-2 (Arbeitskreis OPD-KJ-2, 2020) als Ursache von Symptomen ein intrapsychischer, mindestens seit 6 Monaten andauernder Konflikt angenommen, bei dem unvereinbare Bestrebungen aufeinanderprallen, die in einem aktiven bzw. passiven Modus "gelöst" werden. Sieben Konfliktthemen sind zu unterscheiden: Der NäheDistanz-Konflikt erfasst im aktiven Modus Angst vor Nähe, im passiven Modus dagegen Angst vor Trennung. Beim Konflikt Unterwerfung vs. Kontrolle sind Selbst- und Fremdkontrolle lebensbestimmend und kennzeichnen alle Interaktionen; beim Konflikt Selbst versorgen vs. Versorgtwerden ist in der Interaktion die Versorgung durch materielle und emotionale Güter dominant. Beim Selbstwertkonflikt steht die Regulierung des Selbstwertes im Vordergrund, beim Schuldkonflikt finden sich im aktiven Modus Entwertungen, im passiven Modus zeigen Kinder und Jugendliche mit diesem Konfliktthema eine überzogene Treuebindung an die Eltern. Beim ödipalen Konflikt stehen erotisch-sexuelle Wünsche und deren Abwehr im Vordergrund, beim Identitätskonflikt sind Identitätsfindung und -sicherung lebensbestimmend und färben die
Interaktionen. Entscheidendes Kriterium ist, dass sich der jeweilige intrapsychische Konflikt in verschiedenen Alltagsbereichen beobachten lässt und die Weiterentwicklung des Kindes oder Jugendlichen hemmt oder blockiert.

Der vorliegende Themenschwerpunkt in dieser Ausgabe der Zeitschrift beschreibt zunächst empirische Ergebnisse zur Einstufung der Konfliktachse mit dem Befundbogen nach einem entsprechenden Training mit dem Manual (Arbeitskreis OPD-KJ-2, 2020), stellt aber auch neuere Ansätze vor. Sie beziehen sich zum einen auf Erhebungsmethoden, wie man sie bei jüngeren Kindern, etwa durch eine Story-Stem-Methode, anwenden kann, aber auch auf die Entwicklung und klinische Anwendung eines Einschätzungsverfahrens in Fragebogenform, das in der Selbsteinschätzung für jugendliche Patient_innen und ihre behandelnden Therapeut_innen bzw. Diagnostiker_innen entwickelt wurde. Wegen der konzeptuellen Nähe zwischen dem Strukturniveau und der Ausprägung von Konflikten wird die Assoziation zwischen Konflikt- und Strukturniveau geprüft.

Die in diesem Themenheft vorgestellten Arbeiten stehen im Kontext der Arbeiten zur OPD, dem entsprechenden diagnostischen Instrumentarium für erwachsene $\mathrm{Pa}-$ tient_innen. In den letzten Jahren gab es zahlreiche Forschungsaktivitäten der OPD-E; von 2012 bis 2018 wurden allein 20 englischsprachige Publikationen zur Reliabilität, Validität, zum Einsatz in der Diagnostik und Qualitätssicherung in Langzeitstudien vorgelegt (Both, Basto \& Freitas, 2019). Die OPD hat bereits vor einiger Zeit eine Fragebogenversion, den Konfliktfragebogen, publiziert (Benecke et al., 2018), der u.a. auch im Rahmen der LAC-Depressionsstudie zur Wirksamkeit psychoanalytischer und verhaltenstherapeutischer Langzeitbehandlungen eingesetzt wurde und spezifische Konflikte und Verarbeitungsmodi bei Patient_innen mit Depression ergab (Kaufhold et al., 2017).

Demgegenüber sind die Forschungen zur OPD-KJ erst in den Anfängen begriffen, haben allerdings sowohl zu diagnostischen Fragen des Einsatzes im ambulanten und stationären Setting (z.B. Seiffge-Krenke, Fliedl \& Katzenschläger, 2013a, 2013b) und zur Veränderungsmessung nach stationärer und ambulanter Therapie (z.B. Jelen et al., 2013; Salzer, Cropp, Jaeger, Masuhr \& Streeck-Fischer, 2014) interessante Befunde erbracht. Weitere Forschung ist sinnvoll, um Zusammenhänge etwa der Konfliktachse mit anderen psychodynamischen Konstrukten und weiteren diagnostischen Instrumenten $\mathrm{zu}$ fundieren, um den differenziellen Beitrag dieses Instruments im Vergleich zu anderen Diagnostika abzusichern.

Der spezifische Beitrag der OPD-KJ, und insbesondere der Konfliktachse, im Einsatz in der stationären und ambulanten psychiatrischen und psychotherapeutischen Ver- 
sorgung besteht vor allem darin, über die Diagnose hinausgehende Indikatoren für eine psychische Störung bzw. Beeinträchtigung zu erhalten, die dann therapeutisch gezielt bearbeitet werden können. In der Versorgungsrealität insbesondere bei Jugendlichen hat der überwiegende Anteil der Patient_innen mit psychischen Störungen deutlich mehr als eine Störung gleichzeitig. So beträgt beispielsweise die Komorbidität bei Anorexie zwischen 51 und 73\% (Herpertz-Dahlmann et al., 2001) und die hohe Komorbidität beeinflusst auch den Verlauf (Outcome) bzw. den Therapieeffekt (Bunmi, Cisler \& Tolin, 2010). Es ist also sinnvoll, nach weiteren Indikatoren zu suchen, etwa neben den strukturellen Beeinträchtigungen nach möglicherweise vorhandenen intrapsychischen Konflikten, die die Entwicklung behindern und Symptome „produzieren“. Des Weiteren ist zu beachten, dass bei einer Diagnose, z. B. F93, ganz unterschiedliche intrapsychische Konflikte vorliegen können, die entsprechend bearbeitet werden sollten (Seiffge-Krenke \& Schmeck, 2020).

In diesem Themenheft werden verschiedene Operationalisierungsformen intrapsychischer Konflikte vorgestellt, neben dem klassischen Befundbogen aus dem OPD-KJManual auch eine Methode des Geschichtenerzählens für jüngere Kinder und der Konfliktfragebogen für Jugendliche und ihre Therapeut_innen, der für den Einsatz im ambulanten und stationären Setting der Kinder- und Jugendpsychiatrie bzw. Psychotherapie entwickelt wurde.

In dem Beitrag von Mayer et al. werden Ergebnisse zu intrapsychischen entwicklungsbehindernden Konflikten mit der klassischen Einschätzung durch den Befundbogen im Manual (Arbeitskreis OPD-KJ-2, 2020, S. 390) an zwei Stichproben von klinisch auffälligen Kindern und Jugendlichen, einer ambulanten Psychotherapiestichprobe und einer stationär behandelten Stichprobe in einer Kinder- und Jugendpsychiatrie vorgestellt und zugleich Alters- und Geschlechtsunterschiede in diesen beiden Stichproben analysiert. Intrapsychische entwicklungsbehindernde Konflikte lassen sich bereits bei jüngeren Altersgruppen finden, die diagnostische Erhebung über die Integration von Informationen aus Spiel, Anamnese und Interview ist jedoch recht aufwendig. Es ist daher das Verdienst von König und Benecke, eine für diese Altersgruppe besonders geeignete Methode der Geschichtenergänzung als Teil der OPD-KJDiagnostik entwickelt und überprüft zu haben. In dem Beitrag von Seiffge-Krenke und Escher wird die Entwicklung eines multiperspektivischen Fragebogens vorgestellt, der es erlaubt, intrapsychische Konflikte aus Sicht der Patient innen und ihrer behandelnden Therapeut innen oder Diagnostiker_innen zu erheben. Ein Vergleich zwischen den beiden Methoden (Befundbogen im Manual und Konfliktfragebogen) an einer Trainingsgruppe ergänzt die Entwicklung des Fragebogens. Da Ergebnisse immer auch stichprobenabhängig sind, sollte der Konfliktfragebogen in weiteren Studien erprobt werden. Eine dieser Studien an klinisch auffälligen Jugendlichen wird hier vorgestellt: In dem Beitrag von Cropp und Claaßen geht es um stationäre Patient_innen und ihre Therapeut_innen in einer Kinderund Jugendpsychiatrie.

Generell stellt sich in diesem Kontext die Frage, wie reliabel und valide psychodynamische Konstrukte erfasst werden können und spezifisch inwieweit psychodynamische Konstrukte mit Fragebögen operationalisierbar sind. Frühere Studien hatten erbracht, dass Jugendliche eine recht gute Einschätzung hinsichtlich ihrer Symptome haben (Seiffge-Krenke \& Posselt, 2020). Das spricht dafür, dass die Selbsteinschätzung jugendlicher Patient_innen generell herangezogen werden und eine Ergänzung zum Therapeutenurteil darstellen kann, insbesondere da einige Studien eine Neigung von Therapeut_innen zur Überschätzung der Wirksamkeit ihrer Therapien (Heinonen, 2019) gefunden hatten.

Ganz offensichtlich ist die Einschätzung des Konfliktniveaus bzw. von spezifischen Konfliktthemen für Jugendliche eine anspruchsvolle Aufgabe - nicht nur, weil dies ein sehr hohes Maß an Selbstreflexion und Mentalisierungsfähigkeit bzw. Empathie in andere erfordert, das oftmals nur am Ende der Therapie erreicht ist. Von besonderer Schwierigkeit ist diese Einschätzaufgabe für die Jugendlichen auch deshalb, weil von der grundsätzlichen Ambivalenz der Verarbeitung der Konflikte auszugehen ist, was sich in einem aktiven und passiven Verarbeitungsmodus ausdrückt, und die Art und Weise, wie der Konflikt die Interaktionen mit anderen färbt, der Selbstwahrnehmung nicht so zugänglich ist. Möglicherweise nehmen Therapeut_innen die entwicklungsbehindernden intrapsychischen Konflikten daher deutlicher wahr als dies Jugendlichen am Beginn ihrer Behandlung möglich ist; möglicherweise sensibilisiert die Therapie Jugendliche aber auch für das Vorliegen eines solchen intrapsychischen Konfliktes, sodass sie ihn zunehmend besser wahrnehmen und in der Therapie bearbeiten können. Hier sind noch viele Forschungsfragen offen und dieses Themenheft soll als Anregung dazu dienen, sich eingehender damit zu beschäftigen und die Instrumentarien auszuprobieren. Viele der noch anstehenden Fragen (z.B. Gibt es diagnosespezifische Konflikte? Lassen sich intrapsychische Konflikte durch Psychotherapie verändern? Wie hängen Konflikte und strukturelle Beeinträchtigungen zusammen? Unterscheiden sich Therapeut_innen und Patient_innen in ihrer Wahrnehmung der Effektivität von Psychotherapie zur Veränderung intrapsychischer Konflikte?) lassen sich letztlich nur empirisch beantworten.

Insgesamt zeigt das Themenhaft auf, dass es lohnenswert ist, über die Symptomatik hinausgehende psychodynamische Konstrukte wie Konflikt und Struktur in den diagnostischen Prozess einzubeziehen. Die Ergebnisse zum 
Befundbogen, zu den konfliktspezifischen Geschichtenergänzungsaufgaben als Teil der OPD-Diagnostik im Kindesalter und zum Konfliktfragebogen für Jugendliche und ihre Therapeut innen sind ermutigend und weitere Forschungen zu allen drei Verfahren sind sinnvoll und nötig.

\section{Literatur}

Arbeitskreis OPD-KJ-2. (Hrsg.). (2020). OPD-KJ-2: Operationalisierte Psychodynamische Diagnostik im Kindes- und Jugendalter (3., unveränderte Aufl.). Bern: Hogrefe.

Benecke, C., Henkel, M., Doering, S., Jakobsen, T., Stasch, M., Dahlbender, R. et al. (2018). Der OPD-Konfliktfragebogen. Zeitschrift für Psychosomatische Medizin und Psychotherapie, 64 380-393.

Both, L.M., Basto, A.G. \& Freitas, L.H.M. (2019). Operationalized psychodynamic diagnosis: A systematic review of the literature. Trends in Psychiatry and Psychotherapy, 41, 201-223.

Bunmi, O.O., Cisler, J.M. \& Tolin, D.M. (2010). A meta-analysis of the influence of comorbidity on treatment outcome in the anxiety disorders. Clinical Psychology Review, 30, 642-654.

Dilling, H. \& Freyberger, H.J. (2012). Taschenführer zur ICD10-Klassifikation psychischer Störungen. Bern: Huber.

Heinonen, E. (2019). Was macht einen guten Therapeuten aus? Psychotherapie im Dialog, 20, 18-22.

Herpertz-Dahlmann, B., Müller, B., Herpertz, S., Heussen, N., Hebebrand, J. \& Remschmidt, H. (2001). Prospective 10-year follow-up in adolescent anorexia nervosa - course, outcome, psychiatric comorbidity, and psychosocial adaptation. Journal of Child Psychology and Psychiatry, 42, 603-612.

Jelen-Maubousin, A., Klipsch, O., Pressel, C., Lenz, K., Lehmkuhl, U. \& Winter, S. (2013). OPD-KJ: Veränderungsmessung mit der Strukturachse bei Kindern und Jugendlichen mit psychiatrischer Störung. Psychotherapeut, 58, 24-30.
Kaufhold, J., Negele, A., Leuzinger-Bohleber, M., Kallenbach, L., Ernst, M. \& Bahrke, U. (2017). Zur Konfliktdynamik bei chronischer Depression. Ergebnisse zur Konflikt- und Strukturachse der OPD in der LAC-Studie. Zeitschrift für Psychosomatische Medizin und Psychotherapie, 63, 151-162.

Salzer, S., Cropp, C., Jaeger, U., Masuhr, O. \& Streeck-Fischer, A. (2014). Psychodynamic therapy for adolescents suffering from comorbid disorders of conduct and emotions in an inpatient setting: A randomized controlled trial. Psychological Medicine, 44, 2213-2222.

Seiffge-Krenke, I. (2020). Jugendliche in der Psychodynamischen Psychotherapie: Kompetenzen für Diagnostik, Behandlungstechnik, Konzepte und Qualitätssicherung. Stuttgart: Klett-Cotta.

Seiffge-Krenke, I., Fliedl, R. \& Katzenschläger, P. (2013a). Welche Vorteile bringt eine umfassende Diagnostik mit OPD-KJ bei kinder- und jugendpsychiatrischen Patienten? Zeitschrift für Kinder- und Jugendpsychiatrie und Psychotherapie, 41, 121-132.

Seiffge-Krenke, I., Fliedl, R. \& Katzenschläger, P. (2013b). Diagnosespezifische Strukturdefizite. Konsequenzen für die psychotherapeutische Behandlung von Kindern und Jugendlichen. Psychotherapeut, 58, 15-23.

Seiffge-Krenke, I. \& Posselt, M. (2020). Qualitätssicherung in der psychodynamischen Psychotherapie: Diagnosespezifische Verläufe, der Einfluss unterschiedlicher Informanten und ihre Sicht auf Moderatoren der Veränderung. Zeitschrift für Kinderund Jugendpsychiatrie und Psychotherapie, 49, 19-35.

Seiffge-Krenke, I. \& Schmeck, K. (2020). Diagnostische und therapeutische Arbeit mit der OPD-KJ-2. Göttingen: Vandenhoeck \& Ruprecht.

\section{Prof. Dr. Inge Seiffge-Krenke}

Kostheimer Landstr. 11

55246 Mainz-Kostheim

Deutschland

seiffge-krenke@uni-mainz.de 